\title{
Seminoma: Unusual metastasis involving inguinal lymph node- Review of literature and a case report.
}

\author{
Abhishek Verma ${ }^{1}$, Somya Sinha ${ }^{2}$, Arun Kumar Verma $^{3}$ \\ ' (Department of Laboratory Medicine, Rajendra Institute of Medical Sciences, Ranchi) \\ ${ }^{2}$ (Department of Obstetric and Gynaecology, Seth GS Medical College and KEM Hospital, Mumbai) \\ ${ }^{3}$ (Ex-HOD Department of Pathology, Rajendra Institute of Medical Sciences, Ranchi)
}

\begin{abstract}
We report the case of inguinal Lymphadenopathy in a known and operated case of seminoma. While testicular tumour metastasise to retroperitoneal lymph nodes, metastasis to inguinal nodes is only possible when patient has previous history of testicular or scrotal surgeries. We review the literature to know the incidence of inguinal lymph node involvement in testicular malignancy and discuss possible routes along with metastases to this unusual site. During surgeries minimum handling of the testis and ligation of the spermatic cord up high up to the inguinal ring may reduce metastasis.
\end{abstract}

Keywords: Inguinal lymph node, metastasis, seminoma.

\section{Introduction}

The metastasis of testicular tumors to the inguinal node is rare. Testes drain to retroperitoneal lymph node chain from the level of iliac vessels up to the renal hilum. This is rare and is always associated with previous history of inguinal or scrotal surgery that leads to altered lymphatic drainage.

We present this rare case where an individual who had undergone high inguinal orchiectomy for stage I testicular semiformal, presented with inguinal metastasis.

\section{Case Report}

A 43 years male presented with a left inguinal swelling since 3 months. He was operated outside for left high inguinal orchidectomy for testicular tumors and histopathological diagnosis was seminoma. The tumour makers like alpha feto protein, beta HCG and, lactate dehydrogenase were within limits.. PET- CT revealed $35 \times 45 \mathrm{~mm}$ mass in the left inguinal region, while no lymphadenopathy was noted elsewhere. Patient was subjected to frozen section for diagnosis of this inguinal mass. On frozen section we received a nodal mass which was $3.5 \times 3.5 \mathrm{~cm}$. Cut section of which revealed a well circumscribed yellow coloured area measuring $2 \times 2 \mathrm{~cm}$ surrounded by nodal structure. The most probable diagnosis offered was metastasis of seminoma to inguinal lymph node, in a known and operated case. The histopathology also proved the same diagnosis of seminoma.

\section{Discussion}

The inguinal metastases in a case of seminoma varies from two to ten percentage with a prior history of orchidopexy or scrotal surgery suffering from testicular neoplasm.$^{1,2}$ Daugaard et al. evaluated the inguinal lymph node metastases incidence in 695 patients with stage I testicular cancer ${ }^{1}$. Only two percent of patients had inguinal node metastasis. Inguinal lymph nodes metastasis is seen more commonly in non-seminomatous tumour rather than seminoma. Due to insufficient data available the routine management of the inguinal lymphadenopathy (palpable or not) in patients with testicular tumors and a previous surgery remains controversial. $^{3}$. In some series, patients have been found to have involved inguinal nodes without involved retroperitoneal lymphadenopathy, ipsilateral inguinal lymphadenectomy was performed. ${ }^{4}$ In our case too retroperitoneal nodes were not involved as suggested from imaging studies. Wheeler et al. said that ipsilateral inguinal along with bilateral retroperitoneal node dissection is beneficial in patiensts with nonseminomatous testicular tumor with a previous scrotal and inguinal invasive procedures ${ }^{3}$. In another series where twenty cases of testicular tumor and previous scrotal surgery were presented, did not shows incidence of inguinal lymphadenopathy ${ }^{5}$. They concluded that additional treatment to inguinal nodes is not necessary and patients was given radiation therapy or chemotherapy immediately without nodal removal. Patient with no inguinal lymphadenopathy with history of prior surgery should under go regular groin palpation and dissection of any suspicious lymph nodes was recommended. Involved lymph nodes were to be treated by vinblastine, cisplatinum and bleomycin ${ }^{6}$. Mianne et al. also suggested that prophylactic ipsilateral inguinal dissection is not 
necessary in patients with non-seminomatous testicular tumors with a history of inguinal or scrotal surgery, owing to the efficacy of primary and secondary chemotherapy ${ }^{7}$. However, for testicular seminoma inguinoscrotal radiotherapy is also given. The low incidence of metastasis to inguinal lymph node, morbidity following ilioinguinal radical dissection and the availability of highly successful multimodal therapy makes management an alternative. In our case where only inguinal lymph nodes showed metastasis on frozen section, patient was planned to start with chemotherapy and radiotherapy to reduce further recurrence.

\section{Conclusion}

Inguinal lymph node metastases following testicular tumour is rare. A history of prior inguinal or scrotal surgery leads to involvement of the inguinal nodes due to altered patterns of lymphatic drainage. During inguinal orchidectomy, the surgeon should be careful to minimize the handling of the testis and ensure high ligation of the spermatic cord up to the internal inguinal ring to reduce the risk.

\section{REFERENCES}

[1]. Daugaard G, Karas V, Sommer P: Inguinal metastases from testicular cancer. BJU Int 2006, 97:724-726.

[2]. Batata MA, Whitmore WF Jr, Chu FC, Hilaris BS, Loh J, Grabstald H, Golbey R: Cryptorchidism and testicular cancer. J Urol $1980,124: 382-387$.

[3]. Wheeler JS Jr, Babayan RK, Hong WK, Krane RJ: Inguinal node metastases from testicular tumors in patients with prior orchiopexy. J Urol 1983, 129:1245-1247.

[4]. Johnson DE, Babaian RJ: The case for conservative surgical management of the ilioinguinal region after inadequate orchiectomy. J Urol 1980, 123:44-46.

[5]. Lanteri VJ, Choudhury M, Pontes JE, Wajsman Z, Beckley S, Murphy GP: Treatment of testicular tumors arising in patients with previous inguinal and/or scrotal surgery. J Urol 1982, 127:58-59.

[6]. Ozen H, Altug U, Bakkaloglu MA, Remzi D: Significance of Scrotal Violation in the Prognosis of Patients with Testicular Tumours. BJU 1988,62(3):267-270.

[7]. Mianné DM, Barnaud P, Altobelli A, Masson J, Valeri A: Inguinal lymphatic metastasis of cancer of the testis: staging and therapeutic approach. Ann Urol (Paris) 1991, 25:199-202 